\title{
Synthesis of benzyl and phenethylamines as possible nicotinic receptor antagonists
}

\section{Carolina Méndez ${ }^{1}$, Edwin G. Pérez ${ }^{\star 2,4}$, Bruce K. Cassels ${ }^{3,4}$, Marcos Caroli ${ }^{1}$}

${ }^{1}$ Falcultad de Química y Biología, Universidad de Santiago de Chile, ${ }^{2}$ Facultad de Química, Pontificia Universidad Católica de Chile, ${ }^{3}$ Facultad de Ciencias, Universidad de Chile, ${ }^{4}$ Instituto Milenio de Dinámica celular y Biotecnología *eperezh@uc.cl

Keywords: antagonists, nicotinic receptor, benzylamines, phenylethylamines

\section{INTRODUCTION}

Development of selective agonists or antagonists for the Nicotinic acetylcholine receptors (nAChR) may result in new and potentially useful therapeutic agents. It has been reported in the literature that potent and selective nAChR antagonists could be used for the treatment of nicotine dependence and may be an efficacious adjuvant therapy in many different oncologic protocols. ${ }^{1,2}$ nAChr antagonists studied previously by us having an indole ring core, $N^{1}$-alkyl groups and a quaternized moiety showed potency but little selectivity. ${ }^{3}$
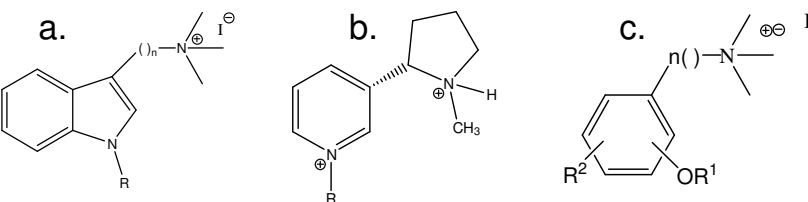

Figure 1. a. alkaloids derivatives Flustra foliacea bryozoo, b. alkyl nicotinic derivatives, c. proposed structures

\section{RESULTS AND DISCUSSION}

O-alkylbenzaldehyde prepared using the Williamson ether synthesis were used to prepare the corresponding benzylamine and phenethylamine.

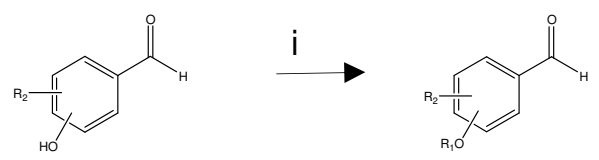

Derivatives of $\mathrm{N}, \mathrm{N}$-dimethylbenzylamine were synthesized by indirect reductive amination.<smiles>C#CCN(CC(=O)c1ccccc1)c1ccc(CC(C)C)cc1</smiles>

Phenethylamine derivatives were synthesized from the reduction of $\beta$-nitroestyrenes previously obtained by Knoevenagel condensation

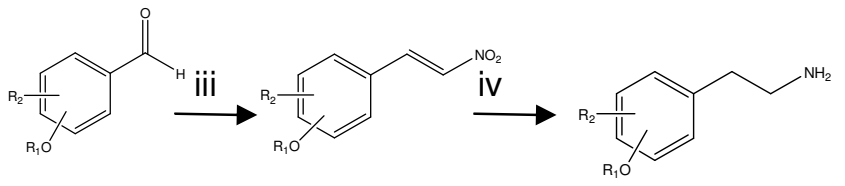

*Conditions: i. $\mathrm{K}_{2} \mathrm{CO}_{3}, \mathrm{EtOH}, \mathrm{Br}$ $\mathrm{C}_{n} \mathrm{H}_{2 n+1}$, reflux 24 h. ii. $\mathrm{Me}_{2} \mathrm{NH}_{2}$, $\mathrm{MeOH}, \mathrm{NaBH}_{4}$. iii. $\mathrm{MeNO}_{2}, \mathrm{AcNH}_{4}$, reflux $10 \mathrm{~h}$. iv. $\mathrm{LiAlH}_{4}$, éther, $24 \mathrm{~h}$. v. $(\mathrm{HCHO}) \mathrm{n}, \mathrm{NaBH}_{4}, \mathrm{ZnCl}_{2}, \mathrm{Cl}_{2} \mathrm{CH}_{2}$.

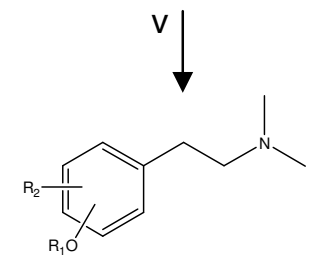

\section{CONCLUSION}

Indirect reductive amination is a good method to obtain substituted benzylamine derivatives of long alkyl chains, the direct method was proven with unsatisfactory results.

The $\beta$-nitroestyrenes reductions was performed using ether as solvent to give better yields compared with those in THF

\section{ACKNOWLEDGEMENTS}

This work was supported by FONDECYT grant 11090120 and ICM grant P05-001-F.

\section{REFERENCES}

${ }^{1}$ Dasgupta, p.; Kinkade, R.; Joshi, B.; DeCook, C.; Haura, E.; Chellappan, S. Proc. Natl. Acad. Sci. U.S.A.., 2006, 103, 6332

${ }^{2}$ Grozio, A.; Peleari, L.; Catassi, A.; Servent, D.; Cilli, M.; Piccardi, F.; Paganuzzi, M.; Cesario, A.; Granote, P.; Mourier, G.; Russo, P. Int. J. Cancer, 2008, 122, 1911.

${ }^{3}$ Pérez, E. P.; Eibl, C.; Gundisch, D.; Cassels B. K. Bioorg. Med. Chem. 2011. Acepted. 